\title{
近年来紫杉醇的合成研究进展
}

\author{
唐 培 $a, b$ 王锋鹏*,b \\ ( ${ }^{a}$ 重庆大学创新药物研究中心 生物工程学院 重庆 401331) \\ $\left({ }^{b}\right.$ 四川大学华西药学院 天然药物化学教研室 成都 610041)
}

\begin{abstract}
摘要 紫杉醇和多烯紫杉醇是目前销量最大的临床抗癌药物. 近年来围绕紫杉醇所进行的高水平研究仍然层出不穷. 综述了 2008 年以来紫杉醇的合成研究进展, 主要涉及紫杉醇 6-8-6 骨架的构建、环系的合成以及侧链的制备.

关键词 紫杉醇; 合成; 研究进展

\section{Recent Progresses in the Synthesis of Taxol}

\author{
Tang, Pei ${ }^{a, b} \quad$ Wang, Fengpeng ${ }^{*, b}$ \\ $\left({ }^{a}\right.$ Innovative Drug Research Centre, Bioengineering College, Chongqing University, Chongqing 401331) \\ ( ${ }^{b}$ Department of Chemistry of Medicinal Natural Products, West China College of Pharmacy, \\ Sichuan University, Chengdu 610041)
}

\begin{abstract}
Taxol and docetaxel are currently the largest selling anticancer drugs. Numerous elegant studies about taxol and its analogues have been reported recently. In this review, the recent progresses in the synthesis of taxol are summarized. These examples cover the synthetic studies of the 6-8-6 tricyclic core, ring system, and side chain.

Keywords taxol; synthesis; progress
\end{abstract}

在药物的研发过程中, 天然产物一直扮演着极其重 要的角色, 而紫杉醇 $\left(\mathbf{1}\right.$, Paclitaxel, Taxol $\left.{ }^{\circledR}\right)($ 图 1)的发现 无疑是其中具有里程碑意义的事件. 围绕紫杉醇所进行 的多学科交叉研究对于天然药物化学、生物化学和药物 开发来说都具有划时代的意义. 迄今紫杉醇和其半合成 类似物多烯紫杉醇 $\left(\mathbf{2}\right.$, Docetaxel, Taxotere $\left.{ }^{\circledR}\right)$ 是历史上销 量最大的临床抗癌药物, 每年的销售额都超过十亿美 金 ${ }^{[1]}$. 它们被广泛的用于卵巢癌、乳腺癌、肺癌以及 Kaposi's 肉瘤的治疗, 而且也在继续进行更多类型肿瘤 的治疗研究以及与其他抗癌药物的联用治疗方法考 察 ${ }^{[2]} .2010$ 年 6 月, 紫杉醇类抗癌药物家族又多了一个 新成员, FDA 批准卡巴他赛 $\left(\mathbf{3}\right.$, Cabazitaxel, Jevtana $\left.{ }^{\circledR}\right)$ 用 于晚期前列腺癌的治疗, 3 即是多烯紫杉醇 $\mathbf{2}$ 的 7,10-二 甲醚产物. 紫杉醇类药物具有独特的抗肿瘤作用机制, 即与 $\beta$-微管蛋白结合后加速蛋白的聚合作用而使微管 稳定, 阻止微管解聚, 使细胞分裂周期停止而导致肿瘤 细胞调亡 ${ }^{[3]}$. 一直以来紫杉醇重要的药理活性、有限的 来源和独一无二的结构骨架吸引着化学家和生物学家
的高度关注, 研究者们围绕紫杉醇类化合物的化学合 成、作用机理和药理活性等进行了大量优秀的研究工作.

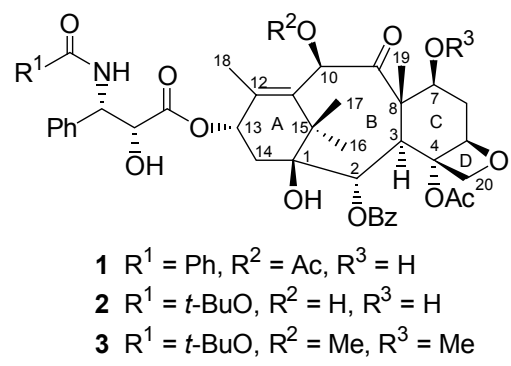

图 1 紫杉醇(1)、多烯紫杉醇(2)和卡巴他赛(3)的结构

Figure 1 Structures of paclitaxel (1), docetaxel (2) and cabazitaxel (3)

由于具有高度官能团化的 6-8-6 骨架结构和复杂的 手性中心, 紫杉醇的合成往往被认为是对化学家最困难 的挑战之一，迄今已有 6 个成功的全合成 ${ }^{[4]}$ 和 1 个形式 合成 ${ }^{[5]}$ 报道, 而研究者们也对紫杉醇的合成进行了详细 的综述 ${ }^{[6]}$. 然而, 对紫杉醇的合成研究却没有因此而停

*E-mail: wfp@scu.edu.cn

Received September 23, 2012; revised November 4, 2012; published online November 5, 2012.

Project supported by the National Natural Science Foundation of China (No. 30873147) and the China Postdoctoral Science Foundation (No. 2012M521670). 国家自然科学基金(No. 30873147)和中国博士后科学基金(No. 2012M521670)资助项目. 
滞，近年来仍吸引着大量学者对其进行合成工作，并报 道了很多精彩而富有特色的环系构建和侧链合成的方 法, 本文详细总结 2008 年以来关于紫杉醇合成研究的 策略和方法.

\section{6-8-6 骨架的构建}

2008 年, Nakada 等 ${ }^{[7]}$ 利用钯催化的分子内甲基酮 $\alpha$ 烯基化反应完成了紫杉烷骨架中八元环的构建. 他们采 用的是汇聚法策略: 首先紫杉醇 $\mathrm{A}$ 环类似物醛 4 与碘代 苯 5 在碱性条件下缩合定量得到单一异构体醇 $\mathbf{6}$, 后经 保护、去保护、氧化和格氏反应等常规反应能高收率生 成关键前体甲基酮 8 , 最后 8 与 $\mathrm{Pd}\left(\mathrm{PPh}_{3}\right)_{4}$ 和 $\mathrm{PhOK}$ 在甲 苯中加热 $24 \mathrm{~h}$ 能以 $96 \%$ 的收率完成关环形成三环化合 物 9 (Scheme 1). 该策略通过 7 步转化以 $65 \%$ 的总收率 制得 6-8-6 三环化合物 9, 但 9 还需通过进一步修饰才能 得到紫杉烷的 6-8-6 骨架.
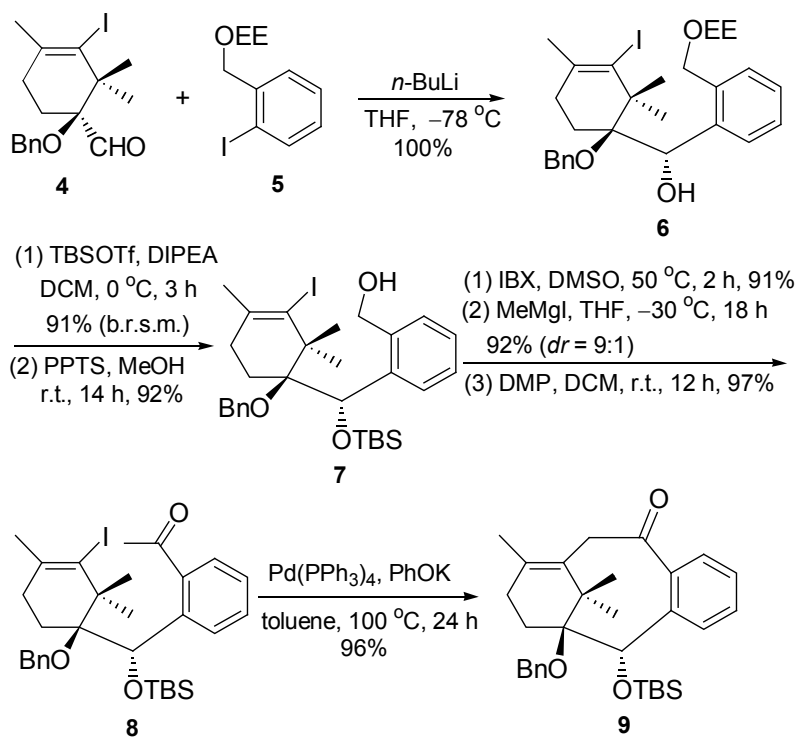

Scheme 1

2008 年, Kakiuchi 研究组 ${ }^{[8]}$ 采用路易斯酸催化下的 重排反应和分子内 Aldol 反应成功构建了紫杉烷 6-8-6 骨架. 环戊烯酮 10 与丙二烯的光加成产物 11 在路易斯 酸 $\mathrm{TiCl}_{4}$ 的催化下发生重排得到烯酮 12 (78\%) (Scheme 2), 12 经过数步高收率的常规反应顺利生成烯 13,13 在 金属钯催化下发生分子内的 Suzuki-Miyaura 偶联反应高 收率 $(85 \%)$ 的得到三环化合物 $\mathbf{1 4}$, 将 $\mathbf{1 4}$ 转化为邻二醇 $\mathbf{1 5}$ 后, 再经 $\mathrm{Pb}(\mathrm{OAc})_{4}$ 氧化和碱性条件下的双键异构化就 以两步共 94\%的产率得到了具有紫杉烷 $\mathrm{AB}$ 环系的双环 二酮 16. 最后进行 $\mathrm{C}$ 环的构建, 将由 $\mathbf{1 6}$ 经多步常规反 应生成的 $\beta$-酮酯 17 与碘代物 18 在碱性条件下发生 $\alpha$ 烷基化反应生成缩醛 19, 紧接着脱除 19 中的缩醛保护 基后在 LDA 作用下顺利发生分子内的 Aldol 反应，以中
等产率 $(56 \%)$ 生成一对等量的难以分离的差向异构体 20, 20 则具有了紫杉烷的 6-8-6环系. 该路线虽然步骤较 多, 但得到的三环产物 $\mathbf{2 0}$ 可方便的用于 $\mathrm{D}$ 环的构筑.

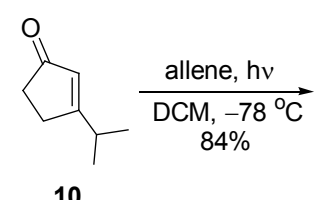

10

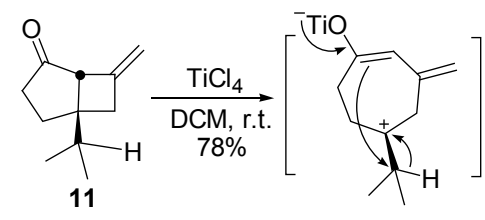

11
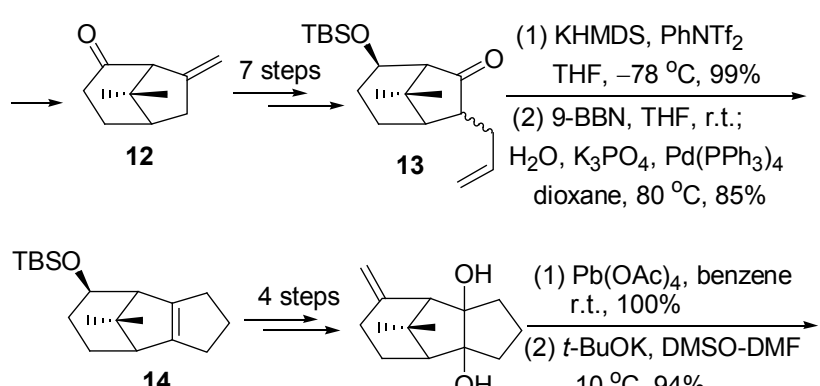

$15 \mathrm{OH} \quad-10^{\circ} \mathrm{C}, 94 \%$

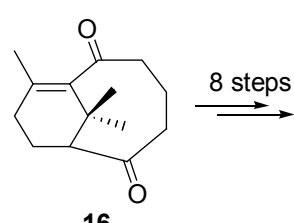

16

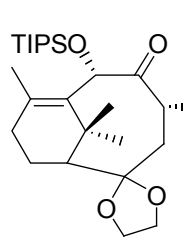

17

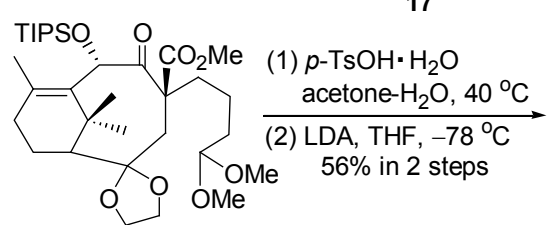

19
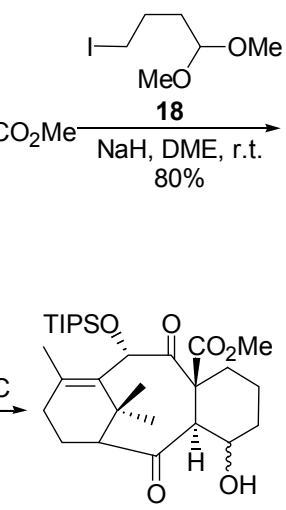

20

\section{Scheme 2}

2008 年, Granja 研究组 ${ }^{[9]}$ 利用二烯炔的串联复分解 关环反应高效的合成了紫杉烷 6-8-6 环系化合物 25a/b. 环已烯酮 21 与烯丙基溴化镁和烯炔醛(S)-22 缩合可定 量制得一对等量的二烯炔异构体 23a/b (Scheme 3), 23a 经还原和保护后得到关键的底物 $24 a / b$, 值得注意的是 当分别采用 DIBAL-H 和 $\mathrm{NaBH}_{4}$ 作为还原剂时, 可以得 到不同比例的产物异构体. 在 Grubbs 第二代催化剂作 用下, 24a/b 可分别发生串联的二烯炔复分解关环反应 生成紫杉烷 6-8-6 三环体系 25a/b, 且收率较高 $(69 \%$ 和 $71 \%$ ). 将 25b 进一步修饰 (Scheme 4): 通过选择性环氧 化和嗍氢化-氧化能高收率的制得环氧醇 $\mathbf{2 7}$, 利用 PDC 将羟基氧化成酩后再用催化量的 $\mathrm{Al}_{2} \mathrm{O}_{3}$ 处理可以促进烯 醇化发生, 从而导致环氧乙烷开环形成位于 $\mathrm{C}(11)$ 和 $\mathrm{C}(12)$ 之间的双键，同时乙酸酕捕获醇盐得到烯酮 28, 最后利用 Luche 条件还原 $\mathbf{2 8}$ 中的不饱和酮基就得到醇 29. 需要指出的是, 另一缩合产物异构体 23b 用上述过 程处理并未得到相应的三环产物, 推测可能是在关环复 
分解反应过程中原料发生分解. 在该方法中, 研究者们 仅通过 5 步反应就可生成 6-8-6 三环产物 25a/b, 且收率 很高(最高可达 61\%).

与上述紫杉醇 6-8-6 骨架构建方法不同的是, 王锋 鹏研究组 ${ }^{[10]}$ 采用天然产物 $\mathrm{C}_{19}$ 二萜生物碱 deltaline (30) 为起始原料, 通过化学转化成功合成了紫杉烷 6-8-6 环 系化合物 36 和 37. Deltaline (30) 经氧去甲基化和 $\mathrm{KMnO}_{4}$ 氧化生成内酰胺 31 (Scheme 5), 接着通过保护 基操作得到的甲基磺酸酯 32 在碱性条件下发生 Grob 裂 解, 然后经甲醚保护就制得了烯酮 33. 臭氧切断 33 中 的双键紧接着发生分子内 Aldol 反应成功构建八元环酮, 然后经甲醚保护以 $70 \%$ 的总产率得到 34 , 与此同时产 生了新的五元半缩醛环. 硼烷还原八元环酮和内酰胺后 得到一对醇的异构体 35, 35 经 Pelletier 裂解顺利生成了 具有紫杉醇 6-8-6 环系的醛 36, 通过进一步修饰就得到 了酮 37.

2009 年, Pattenden 研究组 ${ }^{[11]}$ 利用自由基环化反应成 功合成了紫杉烷 6-8-6 三环骨架. 紫杉醇 A 环类似物 38 与烯基锡化物 39 在 $n$-BuLi 作用下偶联, 接着经 Dess-
Martin 氧化以两步共 $86 \%$ 的产率得到烯酮 40 (Scheme 6), 40 经格氏反应和氧化顺利生成炔 41,41 经碘置换后 用 $n-\mathrm{Bu}_{3} \mathrm{SnH}$ 处理形成烷基自由基，紧接着连续发生 12-endo-dig 和 6-exo-trig 的两次成环过程就以中等收率 (44\%)得到 6-8-6 三环化合物 43. 对于复杂多环天然产 物的合成来说，这种简捷的串联自由基环化反应无疑是 一种非常有用的策略，但总收率相对较低(14\%).

2011 年, Suffert 研究组 ${ }^{[12]}$ 报道了一种通过过渡金属 钯催化的 Domino 反应简捷快速的构建紫杉烷 6-8-6 骨 架结构的方法(Scheme 7). 以 2-溴环已烯酮 44 为起始原 料, 经常规反应制得炔烃 45,45 与醛 46 在 $n$-BuLi 作用 下得到关键前体丙炔醇 47 (60\% 90\%), 在微波作用下 47 经钯催化的 Domino 反应可以 $23 \% \sim 65 \%$ 的产率一步 合成四环产物 $48 a / b$, 将 $48 a / b$ 通过 Dess-Martin 氧化顺 利制得烯酮 $49 a / b$. 而在接下来关键的选择性双键氧化 裂解反应中, 顺式产物 49a 表现出了很好的选择性, 可 得到具有多官能团的紫杉烷 6-8-6 三环产物 50 (19\% $62 \%$ ), 但反式产物 49b 可能由于选择性差而导致反应非 常复杂. 该策略十分高效，仅通过 5 步反应就可生成 6-<smiles>O=C1C=CCCC1</smiles>

21
(1) AllMgBr, $\mathrm{CuBr}$ $\mathrm{LiCl}, \mathrm{THF},-78^{\circ} \mathrm{C}$

(2)

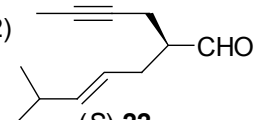

(S)-22 THF, $-78^{\circ} \mathrm{C}$ $100 \%$ in 2 steps

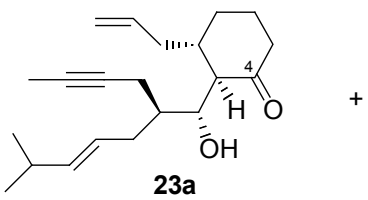

23a

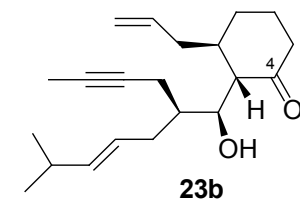

23b

1) DIBAL-H, DCM, $-78{ }^{\circ} \mathrm{C}, 76 \%, \mathbf{2 4 a}: \mathbf{2 4 b}=1: 2$

(2) $\mathrm{NaBH}_{4}, \mathrm{MeOH}, 89 \%, \mathbf{2 4 a}: \mathbf{2 4 b}=7: 1$

(3) MEMCI, DIEA, DCM, r.t., $85 \%$ 96\%

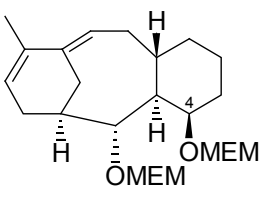

25a

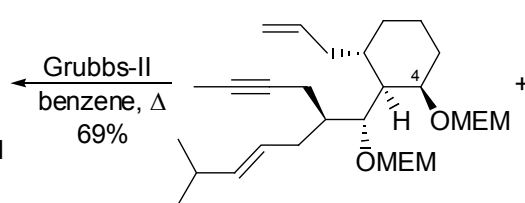

24a

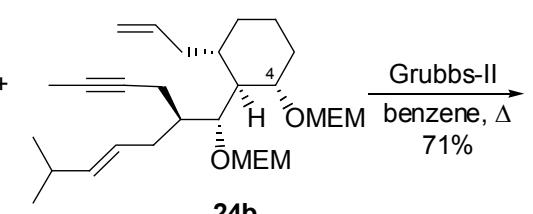

24b

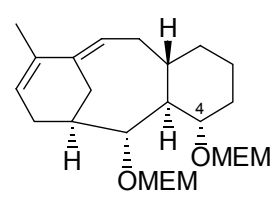

25b

Scheme 3

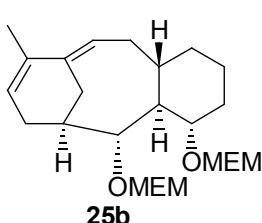

25b
$m$-CPBA $\mathrm{DCM}, 0{ }^{\circ} \mathrm{C}$ $93 \%$

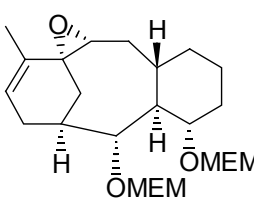

26

\section{$\underset{\mathrm{BH}_{3}-\mathrm{THF}, 0{ }^{\circ} \mathrm{C}}{\mathrm{H}_{2}, \mathrm{NaOH}}$
$\underset{96 \%}{\longrightarrow}$}

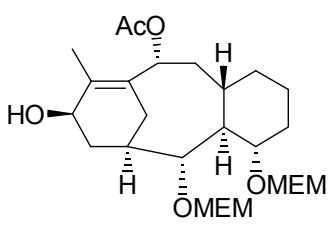

29

Scheme 4 


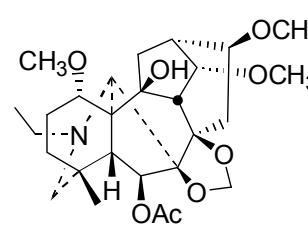

30

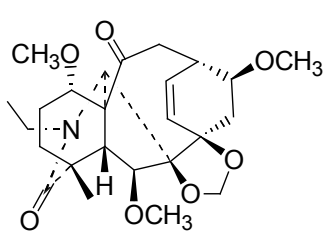
33

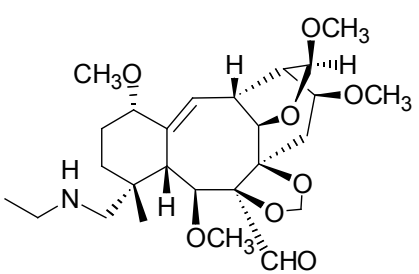

36

||<smiles>C=O</smiles>

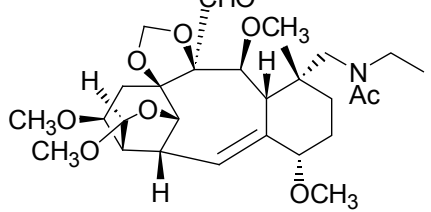

(1) $\mathrm{Ac}_{2} \mathrm{O}$, pyridine (2) $m$-CPBA, DCM

(3) $\mathrm{HOAc} / \mathrm{H}_{2} \mathrm{O}$ $63 \%$ in 3 steps

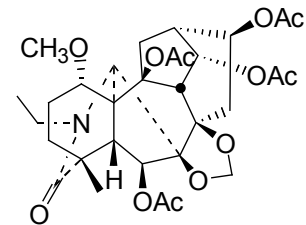

31

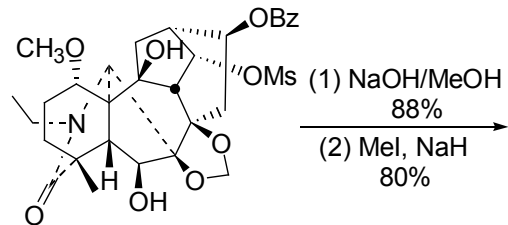

32 \begin{tabular}{l} 
(1) $\mathrm{NaOH} / \mathrm{MeOH}$ \\
(2) $\mathrm{BzCl}, \mathrm{Ag}_{2} \mathrm{O}, \mathrm{KI}$ \\
\hline $\begin{array}{c}\text { (3) } \mathrm{MsCl}, \text { pyridine } \\
90 \% \text { in } 3 \text { steps }\end{array}$
\end{tabular}

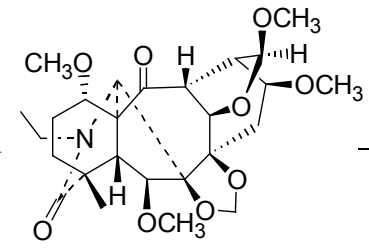

34

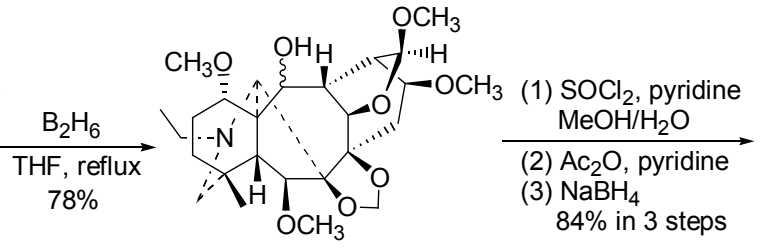

35

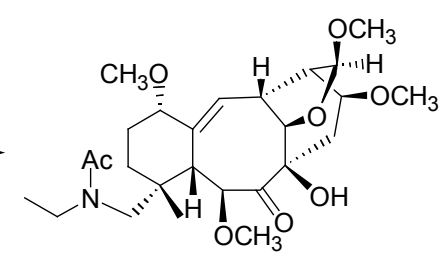

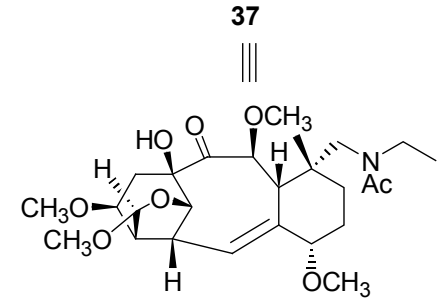

Scheme 5

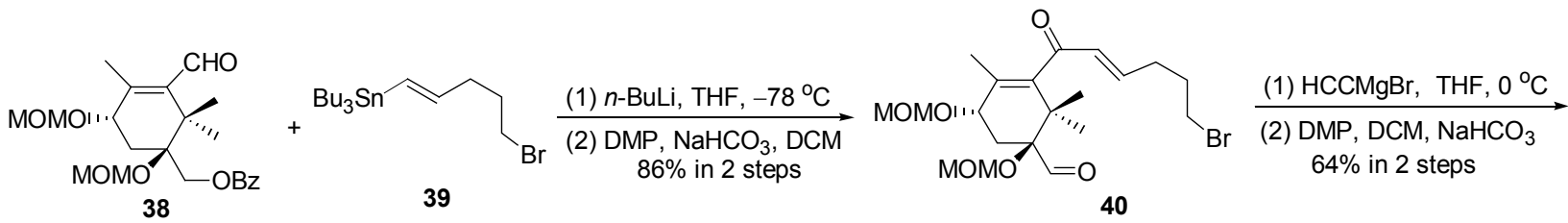<smiles>C#CC(=O)[C@@]1(C)C(C(=O)/C=C/CCCBr)=C(C)[C@H](OC)C[C@H]1OC</smiles>

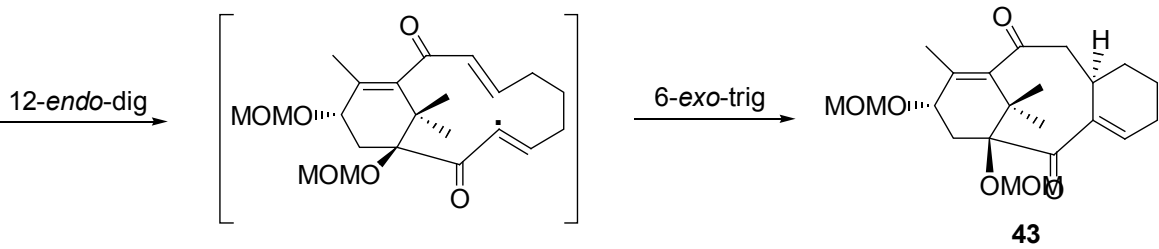

Scheme 6

8-6 三环产物 50. 而在关键的钯催化成环反应中，同时 形成了三个新的碳碳键、两个立体中心和三个碳环, 作 者也推测了反应的机理: 首先丙炔醇 47 经氧化加成和 两次环化过程(4-exo-dig 和 6-exo-trig)形成新的三环体系
(Scheme 8), 紧接着分别经顺式和反式脱氢去钯化消除 和电环化分别得到顺式和反式四环产物 $48 \mathrm{a}$ 和 $48 \mathrm{~b}$.

最近, Baran 研究组利用不对称 Michael 加成、 Mukaiyama-Aldol 缩合和分子内 Diels-Alder 反应等关键 
<smiles>O=C1CCCC=C1Br</smiles>

44<smiles>C=C=C1OC(C)(C)OC12CCCC=C2Br</smiles>

45

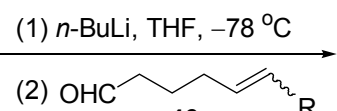

46

$60 \% \sim 90 \%$<smiles>[R]C=CCCC[C@@H](O)C#C[C@H](OC(C)(C)C)[C@@]1(O)CCC[C+]=C1Br</smiles>

$\mathrm{Pd}\left(\mathrm{PPh}_{3}\right)_{4}, i-\mathrm{Pr}_{2} \mathrm{NH}$, benzene

microwave, $160{ }^{\circ} \mathrm{C}, 20 \mathrm{~min}$ $23 \% \sim 65 \%$

$\mathrm{R}=\mathrm{H}, \mathrm{SiEt}_{3}, \mathrm{SiMe}_{3}, \mathrm{Ph}, 4-\mathrm{MeOC}_{6} \mathrm{H}_{4}, 4-\mathrm{CF}_{3} \mathrm{C}_{6} \mathrm{H}_{4}, \mathrm{CO}_{2} \mathrm{Me}$

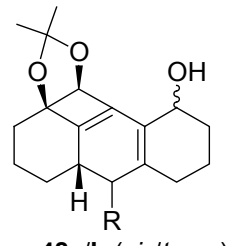

48a/b (cis/trans)

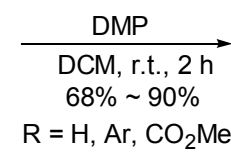

$\mathrm{R}=\mathrm{H}, \mathrm{Ar}, \mathrm{CO}_{2} \mathrm{Me}$

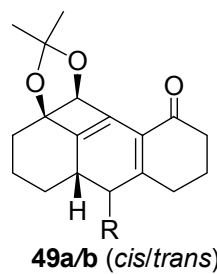

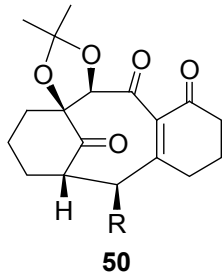

Scheme 7

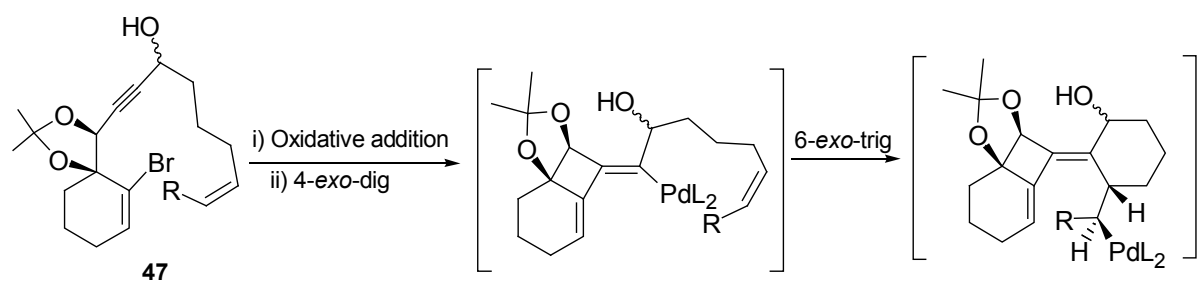

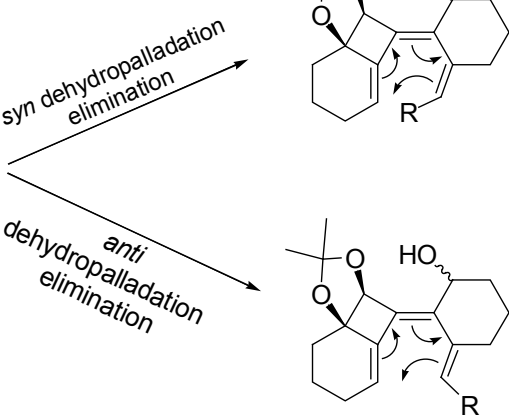

$6 \pi$ eletrocyclization

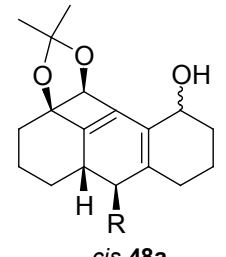

$6 \pi$ eletrocyclization

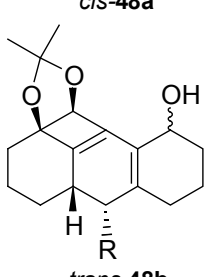

Scheme 8

策略，简洁高效的完成了紫杉醇 6-8-6 环系化合物 59 和 60 的对映选择性合成，从而为紫杉醇的全合成打下了 坚实的基础. 分别从商品化原料 51 和 52 出发(Scheme 9), 参照文献方法顺利制得溴化物 $\mathbf{5 3}$ 和烯烃 $\mathbf{5 4}$. 然后 53 和 54 在路易斯酸的促进下发生有机铜催化的 1,6-加 成, 以 $86 \%$ 的收率生成烯酮 $\mathbf{5 5}$, 这步反应操作简便, 并 且每次可以达到十克级的规模. 接着在噻吩-2-甲酸亚 铜和手性亚磷酰胺配体 56 的作用下, 55 与三甲基铝顺 利发生不对称 Michael 加成, 在 C(8)引入甲基形成手性 季碳中心从而得到关键的烯醇硅醚 $\mathbf{5 7}$, 该反应表现出 了非常好的对映选择性(93\%)和收率(89\%). 在接下来的 Mukaiyama-Aldol 反应中, 作者在尝试了多种路易斯酸 后发现, 仅 $\operatorname{Gd}(\mathrm{OTf})_{3}$ 能使丙烯醛和 57 顺利缩合, 然后
经 Jones 氧化生成一对难以分离的烯酮异构体 58 (85\%, $d r=2: 1$ ), 将此混合物用 $\mathrm{BF}_{3} \cdot \mathrm{OEt}_{2}$ 处理, 顺利发生非对 映选择性的 Diels-Alder 反应以 47\%的收率得到 6-8-6 三 环物 59, 以及相应的 C(3) 异构体 (29\%). 最后，经烯醇 化和 Negishi 偶联就可以生成(+)-taxadienone (60), 60 是 合成紫杉烷类化合物的关键中间体. 该合成路线总共仅 有 8 步化学操作，而总产率高达 18\% 20\%, 并且每步 反应均能进行成克级的制备, 如此漂亮的合成路线也为 利用全合成方法对紫杉醇进行工业化生产提供了可能 性.

\section{2 环系的合成}

2008 年, Kaliappan 等 ${ }^{[14]}$ 采用烯炔交叉复分解/分子 


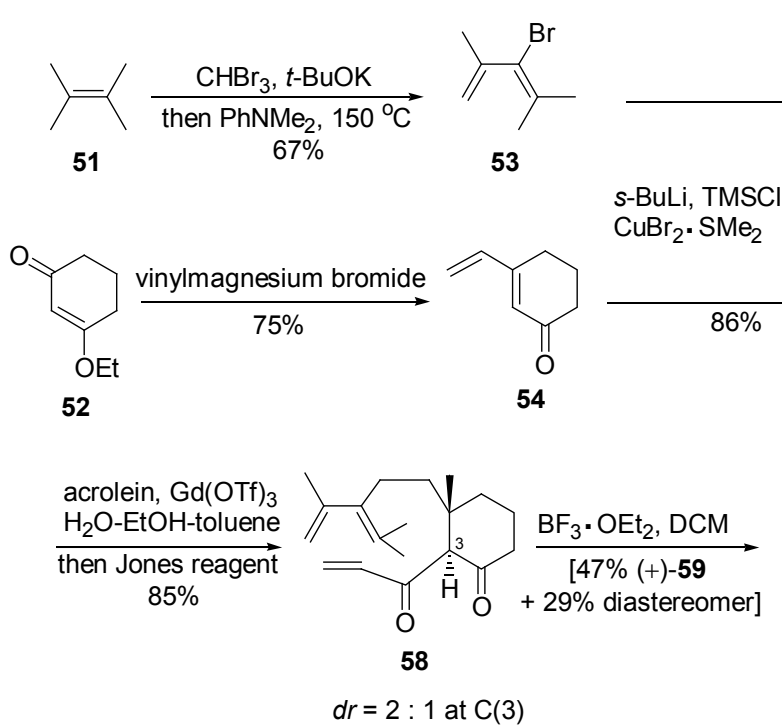

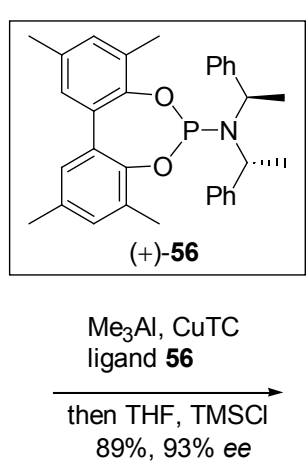

55

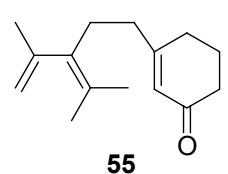

$89 \%, 93 \%$ ee

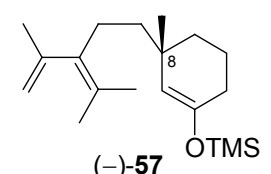

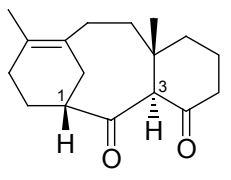

(+) -59

$d r>99: 1$ at $\mathrm{C}(1)$

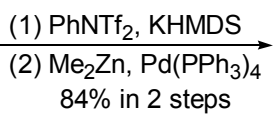

$\mathrm{Me}_{2} \mathrm{Zn}, \mathrm{Pd}\left(\mathrm{PPh}_{3}\right)$
$84 \%$ in 2 steps

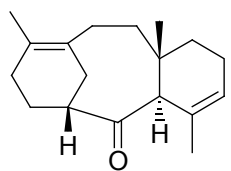

$(+)-60$

Scheme 9

内 Diels-Alder (IMDA)串联反应快速构建了紫杉烷 $\mathrm{AB}$ 环系 63. 以 $D-(+)$-核糖缩酮物 61 为起始原料, 经多步 转化得到关键的串联反应前体烯炔 62. 在 Grubbs 第二 代催化剂作用下, 62 与乙烯发生烯炔交叉复分解反应后 紧接着完成分子内的 Diels-Alder 反应(IMDA), 以 $62 \%$ 的收率关环得到具有紫杉醇 $\mathrm{AB}$ 环的酮 63 (Scheme 10).

同年, Prunet 等 ${ }^{[15]}$ 报道了利用烯烃关环复分解反应 构建紫杉烷 BC 环系的方法, 并对 C(1),C(2)-二醇的不同 取代情况做了详细研究. 为了制备复分解反应的前体, 他们用消旋的醛 64 与光学纯的腙 65 发生 Shapiro 偶联 得到一对邻二醇的非对映异构体 66a/b (Scheme 11), 接 着将去保护后的三醇 $67 \mathbf{a} / \mathbf{b}$ 用 Grieco 所报道的方法处理 并用碳酸酯保护邻二醇就以中等收率生成了二烯 $69 \mathrm{a} / \mathrm{b}$. 通过与不同的 Grubbs 催化剂反应, 发现只有与紫杉醇 $C(1), C(2)$ 取代构型相同的 69b 能发生理想的烯烃关环 复分解反应得到紫杉烷 $\mathrm{BC}$ 环系类似物 70b, 而有着相 反构型的 69a 没有获得目标产物. 类似的, 在缩酮保护 邻二醇和裸露邻二醇的情况下, 也仅是构型相符的前体 $71 \mathrm{~b}$ 和 73b 可分别发生烯烃关环复分解反应定量的得到 目标产物 72b 和 74b (Scheme 12). 而有趣的是, 当 C(2)
为苯甲酰基取代时, 两种构型的前体 75a/b 均能成功关 环得到 $\mathrm{BC}$ 环产物 $\mathbf{7 6 a} / \mathbf{b}$. 作者通过分子模型比较, 认为 各种前体中的 $\mathrm{C}(10)$ 与 $\mathrm{C}(11)$ 之间的距离是决定能否发 生复分解关环反应的重要因素.

在已报道的紫杉醇的全合成中, Danishefsky 路线是 唯一一个将 $\mathrm{CD}$ 环的合成作为全合成开端的策略 ${ }^{[\mathrm{c}, 4 \mathrm{~d}]}$, 2008 年, Audran 研究组 ${ }^{[16]}$ 完成了另一高效和立体选择 性的 Danishefsky CD 环中间体 $(+)-84$ 的合成. 作者以光 学纯的内酯 $(+)-77$ 为起始原料(Scheme 13), 经烯烃的 氧化切断、Wittig 反应和缩醛保护得到 $(+)-\mathbf{- 7 8}$, 接着将 其内酯还原可高收率的得到二醇(-)-79. (一)-79 经羟基 保护和立体选择性的烯丙位氧化可顺利制得醇 $(+)-80$, 然后经氧化及一系列的保护基操作就生成了 D 环关环 的前体甲磺酸酯 81. 在 DBU 作用下, 81 可顺利发生亲 核取代反应而关环生成环醚(一)-82, 最后经叔醇的芐醚 保护、伯醇的去保护和氧化就得到了 Danishefsky CD 环 片断(+)-84. 显然, 与 Danishefsky 合成 $(+)-84$ 的方法 (24 步, 总收率 5\%)相比, Audran 的策略无论是在反应步 骤(15 步)还是总收率(11.4\%)上都大大的优化了.
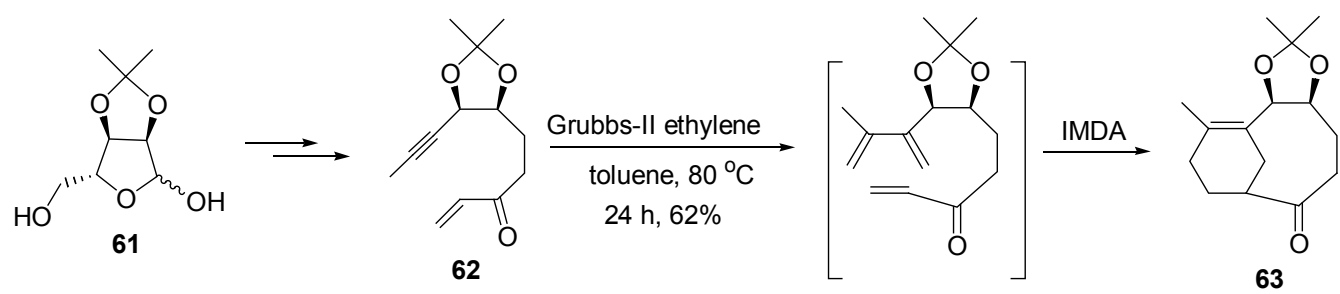

Scheme 10 

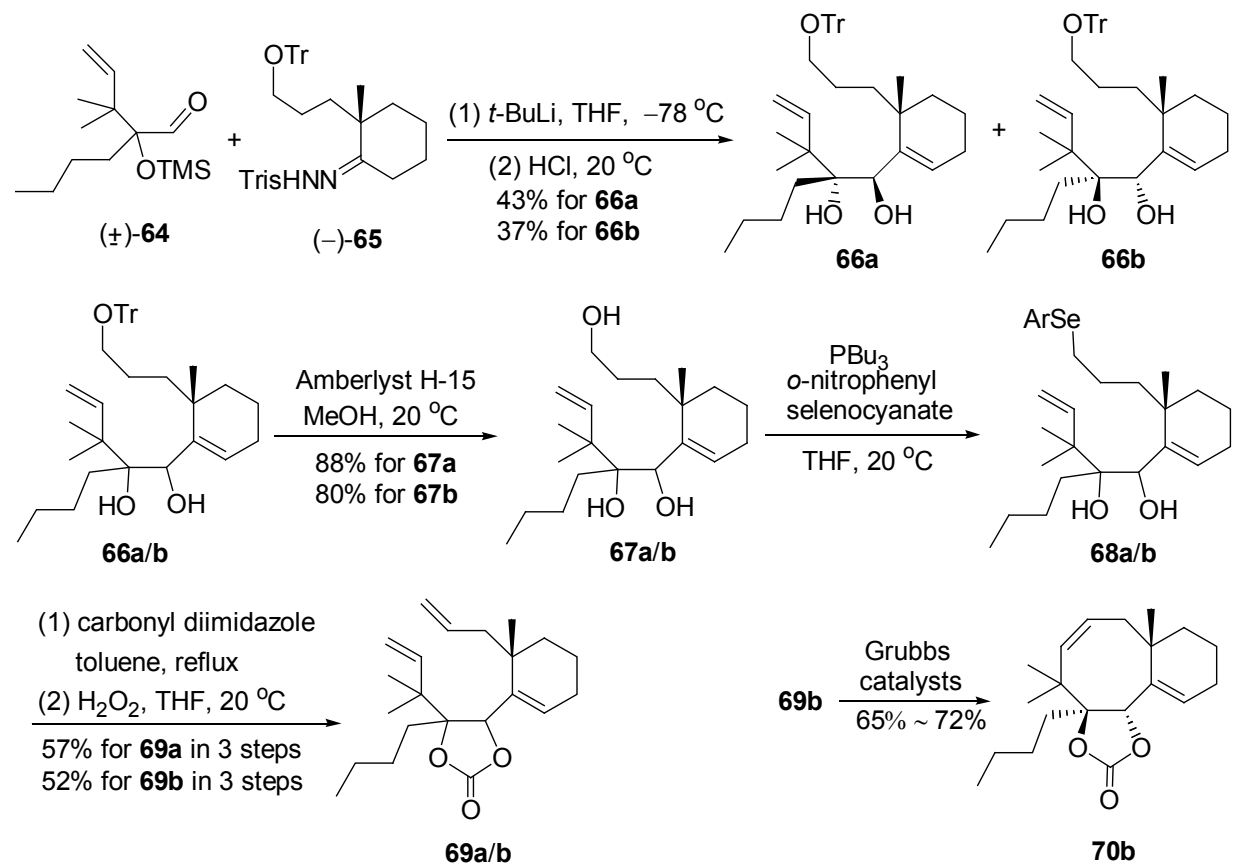

Scheme 11

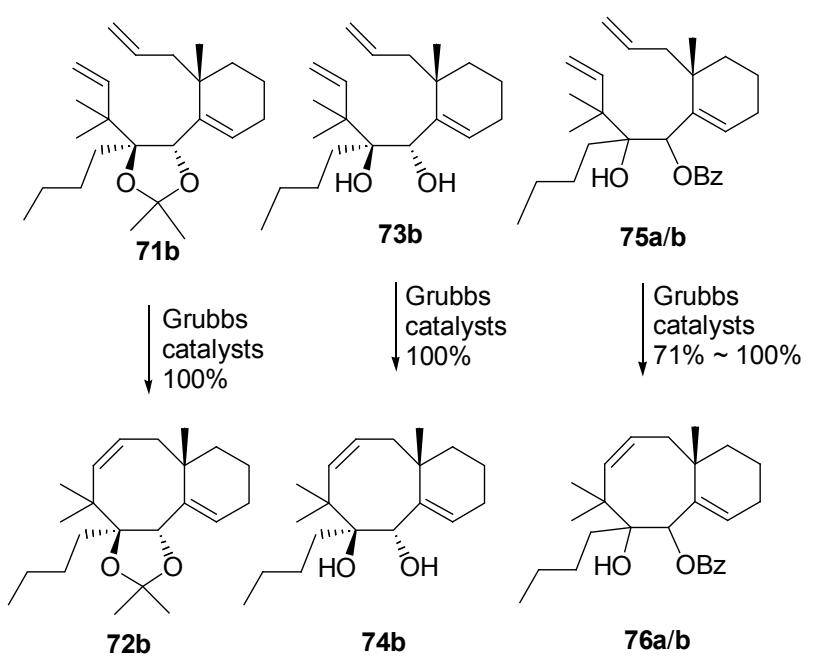

Scheme 12

2009 年, Takahashi 等 ${ }^{[17]}$ 报道了立体选择性的三组 分一锅煮合成紫杉烷 $\mathrm{AC}$ 环系的方法. 羟基氰酯 85 与含 取代的环已烯醞 86 发生 1,4-加成, 产生的烯醇盐立即被 体系中的第三组分甲醛捕获, 即生成了具有 $\mathrm{AC}$ 环系的 三组分偶联产物 87 (Eq. 1, Scheme 14), 收率最高可达 $90 \%$.

2011 年, Prunet研究组 ${ }^{[18]}$ 以 Wieland-Miescher 酮(89) 为起始原料, 完成了紫杉醇 $\mathrm{C}$ 环类似物的对映选择性合 成. 首先, 在 $\mathrm{NaBH}_{4}$ 和(一)-TADDOL 的作用下, Wieland-Miescher 䣯(89) (70\% ee) 被还原生成光学纯的醇 90 ( $99 \%$ ee) (Scheme 15). 90 经多步常规反应可得到紫杉醇 $\mathrm{C}$ 环类似物 94, 但将 94 转化为腙的尝试没有成功. 接着
将 90 中的羟基用苯甲酰基保护后，再经臭氧化和酯化 得到甲酯 96 (Scheme 16), 此时可顺利将 96 转化为腙进 而制得可用于 Shapiro 偶联的 C 环前体缩醛 99. 然而, 该路线中将 97 还原成 98 时收率不高(49\%), 为此作者再 次优化了合成策略: 90 经硅醚保护和臭氧化反应生成酸 101 (Scheme 17). 接着利用 DMF 和草酰氯作用产生的 Vilsmeier-Haack 试剂将酸 101 还原成醛，同时完成关环 生成一对等量的异构体混合物 102 , 半缩醛 102 经甲醚 保护后则顺利制得目标化合物腙 104, 该路线总收率为 $24 \%$, 但步骤相对较多(共 7 步). 此外, 作者还进行了 Shapiro 偶联的模拟反应: 104 与苯丙醛在碱性条件下顺 利发生 Shapiro 反应，以 $42 \%$ 的收率生成了偶联产物 105, 这表明 104 是有效的 C 环前体.

\section{3 侧链的制备}

由于资源的限制和全合成的制约，现阶段紫杉醇类 抗癌药的来源主要是靠半合成手段, 即用易得的 10-去 乙酰巴卡亭 III (10-DAB)与侧链缩合得到成品. 因此, 高效的合成具有光学活性的紫杉醇侧链一直以来都是 学术界和工业界的研究热点.

2009 年, Mustafa 等 ${ }^{[19]}$ 以易得的手性环氧酯 106 为 原料(Scheme 18), 先后与叠氮化钠和苯甲酰氯反应生 成叠氮物 107, 107 经 $\mathrm{Zn}-\mathrm{TMSCl}$ 作用下的还原及酰基迁 移以 $58 \%$ 的收率得到酰胺 108,108 经脱保护就制得了光 学纯的紫杉醇侧链 109. 这样从 106 出发通过 4 步转化 以 $18 \%$ 的总收率就可得到紫杉醇侧链. 

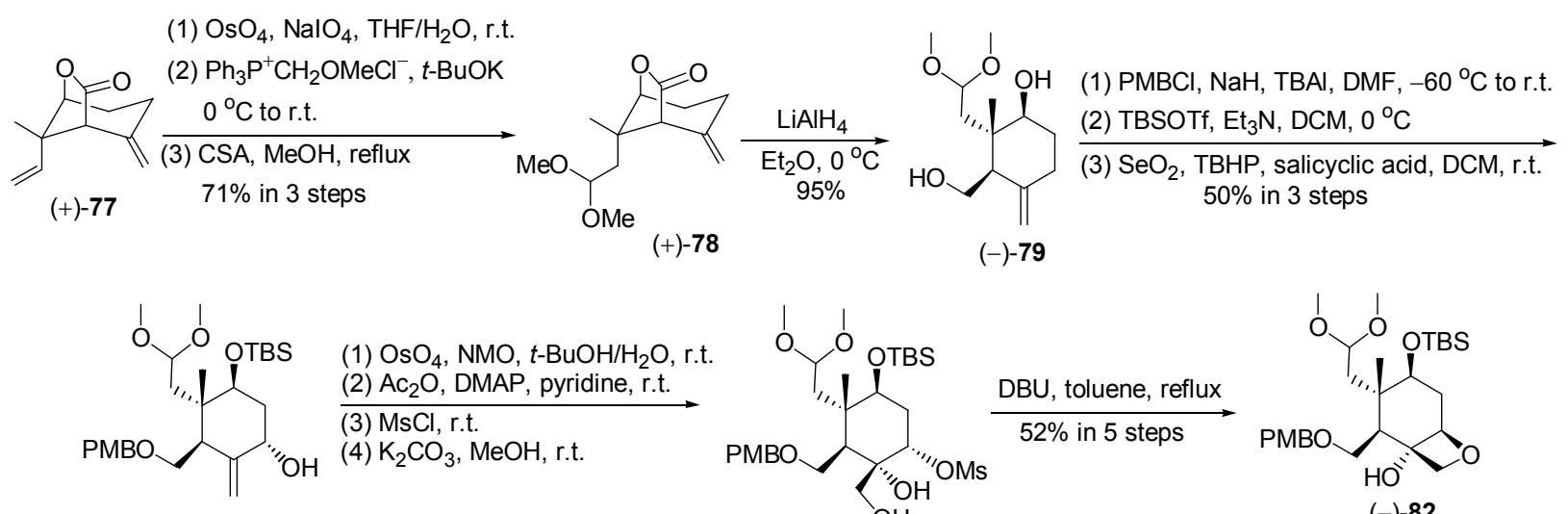

$(+)-80$

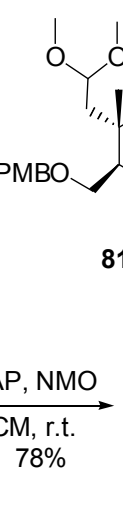

I I OTBS

(2) TBSOTf, $\mathrm{Et}_{3} \mathrm{~N}, \mathrm{DCM}, 0^{\circ} \mathrm{C}$

$50 \%$ in 3 steps

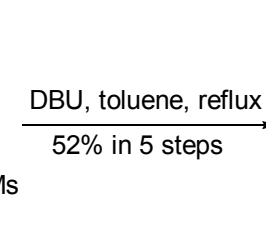

81

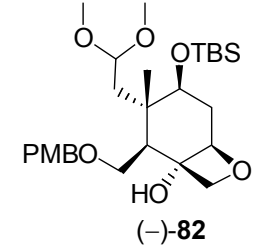

$(-)-82$ $\frac{(1) \mathrm{BnBr}, \mathrm{NaH}, \mathrm{TBAl}, \mathrm{THF} \text {, r.t. }}{\text { (2) } \mathrm{DDQ}, \mathrm{DCM} / \mathrm{H}_{2} \mathrm{O}, 0{ }^{\circ} \mathrm{C} \text { to r.t. }}$
$83 \%$ in 2 steps

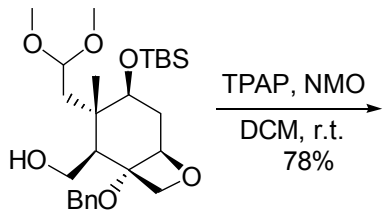

$(+)-83$
OTBS

(+)-84

Scheme 13

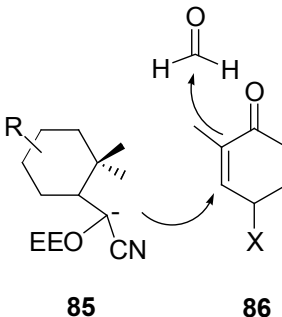
$\underset{\text { 3-component coupling }}{\stackrel{\text { conditions }}{\longrightarrow}}$

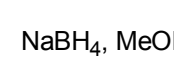

$\mathrm{NaBH}_{4}, \mathrm{MeOH}$

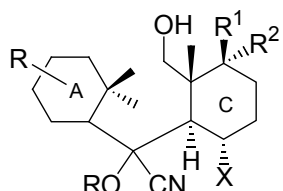

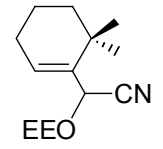

$85 a$
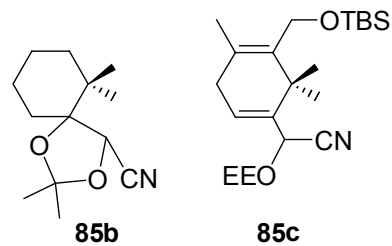

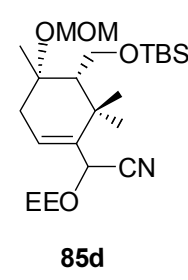

$85 d$
87: $R^{1}=R^{2}=0$

88: $R^{1}=O H, R^{2}=H$ up to $90 \%$ yield

\section{Scheme 14}<smiles>CC12CCC(=O)C=C1CCCC2=O</smiles>

89

$70 \%$ ee<smiles>CO[C@H]1CCCC(=O)[C@]1(C)CCC(=O)O</smiles>

92

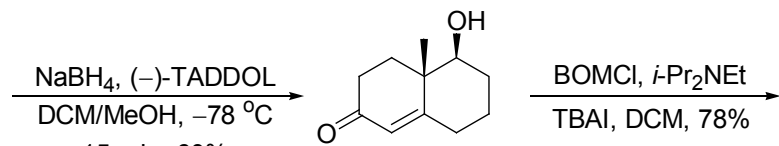

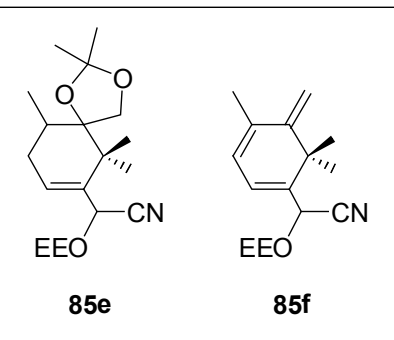

90

$99 \%$ ee

$15 \mathrm{~min}, 60 \%$

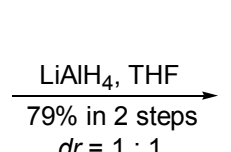
$d r=1: 1$<smiles>CO[C@H]1CCC[C@@H](O)[C@]1(C)CCCO</smiles>

93<smiles>CO[C@H]1CCCC2=CC(=O)CC[C@]2(C)C1(C)O[Na]</smiles>

91

Scheme 15 

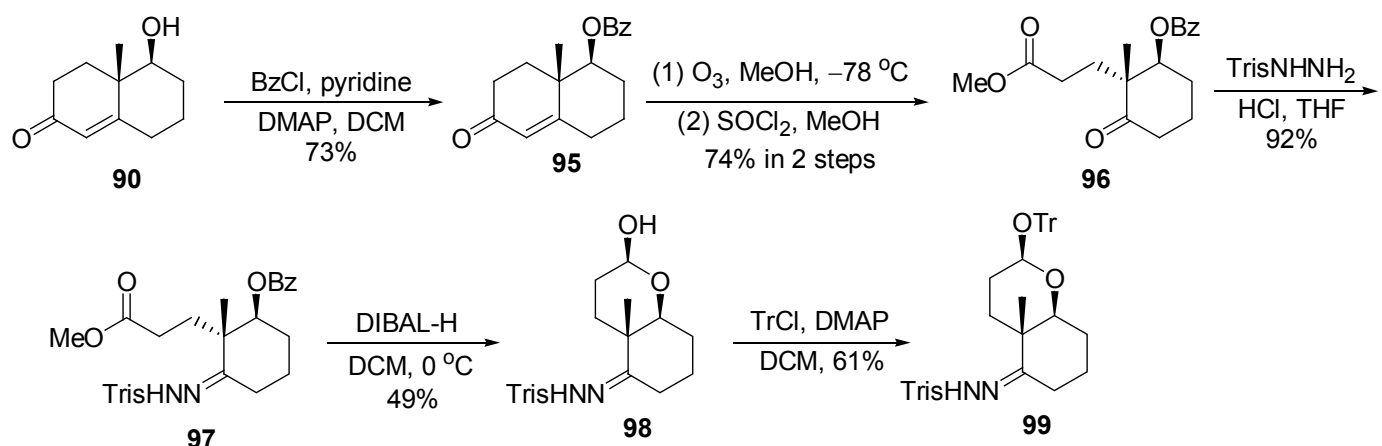

\section{Scheme 16}

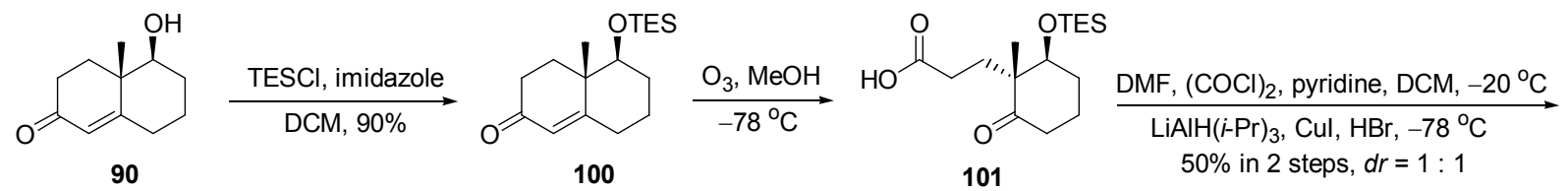<smiles></smiles>

102<smiles>COC1CCC23CCCCC2OC(=O)CCC3O1</smiles>

103

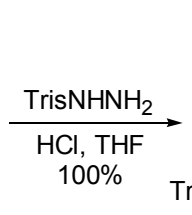

TrisH

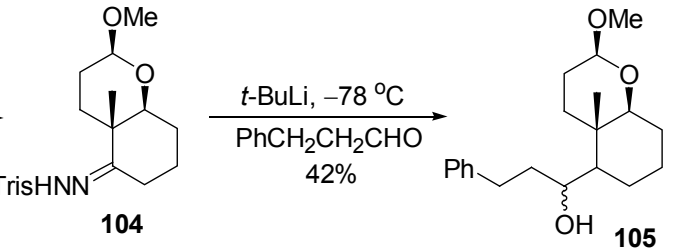

Scheme 17<smiles></smiles>

(1) $\mathrm{NaN}_{3}$, ethylformate $\mathrm{DMF}, 60^{\circ} \mathrm{C}$

(2) $\mathrm{BzCl}, \mathrm{DMAP}$ DCM, r.t. $49 \%$ in 2 steps

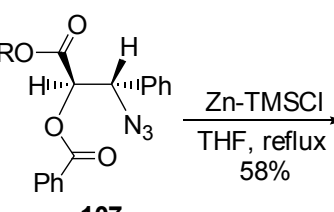

107

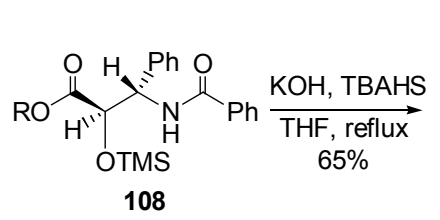<smiles>O=C(N[C@H](c1ccccc1)[C@H](O)C(=O)O)c1ccccc1</smiles>

Taxol Side Chain

\section{Scheme 18}

Córdova 等 ${ }^{[20]}$ 发现在 $(R)$-脯氨酸的催化下 (Scheme 19), 苯酰胺 110 和醛 111 可发生高对映选择性的缩合得 到醛 112, 112 经氧化成酸就得到了紫杉醇侧链的前体化 合物 113. 该方法简洁高效, 对映选择性高, 总收率为 56\%, 而且产物 113 仅需脱除苄基就可方便的生成紫杉 醇侧链.

$$
\text { (R)-proline (20 mol\%) }
$$

\section{Scheme 19}

Soderquist 研究组 ${ }^{[21]}$ 在研究不对称有机硼反应时, 发现利用 10-三甲基硅-9-嗍双环 [3.3.2]癸烷(10-TMS-9-
BD)体系可对反应进行高效的立体控制，他们也将这一 策略应用于紫杉醇侧链类似物的合成中. 10-TMS-9BBD 体系 $\mathbf{1 1 4}$ 与醛亚胺 $\mathbf{1 1 5}$ 反生不对称的 $\gamma$-甲氧基烯丙 基化反应，生成有机嗍化合物 116，再依次用酸和碱处 理后则以较高的对映选择性得到苯胺 117 (Scheme 20), 117 经苯甲酰化和氧化就制得了紫杉醇侧链衍生物 118 . 
2010 年, 胡文浩研究组 ${ }^{[22]}$ 报道了利用手性 Brønsted 酸氧膦配体和 $\mathrm{Rh}_{2}(\mathrm{OAc})_{4}$ 联合催化进行的对映选择性三 组分反应合成紫杉醇侧链 109. 重氮乙酸酯 119、芳香醇 120 和亚胺 121 三组分在 $\mathrm{Rh}_{2}(\mathrm{OAc})_{4}$ 和氧膦配体催化下, 对映选择性的生成酯 122 (Scheme 21), 然后经甲酸铵 /Pd-C 氢化脱市和重结晶后能以中等收率和高对映选择 性的得到 $\alpha$-羊基酯 123. 最后采用常规的硅醚保护、苯 甲酰化和去保护操作成功得到紫杉醇侧链 109. 该合成 方法虽然步骤较多(7 步转化), 总收率较低(12\%), 但通 过有机催化和重结晶操作可获得很好的对映选择性.

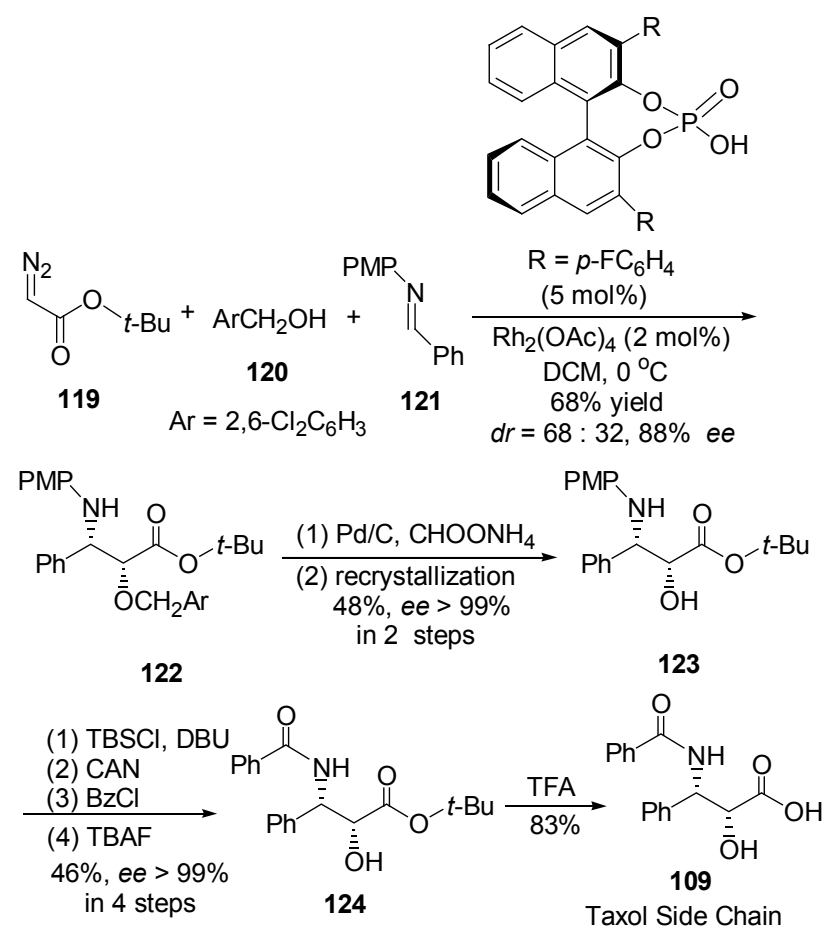

Scheme 21

在紫杉醇侧链的合成研究中, 酶促反应也得到了很 好的应用. 最近, Fülöp 等 ${ }^{[23]}$ 利用酶促反应高对映选择性 的合成了紫杉醇侧链的关键中间体 (2R,3S)-3-苯基异丝 氨酸 (一)-129 (Scheme 22). 将酰氯 125 与亚胺 126 缩合 形成 $\beta$-内酰胺 127, 将由 127 水解得到的消旋体氨基酯 128 用脂肪酶 PS-IM 处理, 可以 $47 \%$ 的收率得到光学纯 的酯(一)-128, 然后将其水解就生成了紫杉醇侧链中间 体(一)-129. 或者将内酰胺 127 用脂肪酶 CAL-B 处理可 发生串联的酶促反应直接得到光学纯的(-)-129.

\section{4 总结与展望}

自从 1967 年被发现开始, 几十年来紫杉醇一直受 到众多研究者的亲睐, 化学、生物及药物研发领域的学 者们对其进行了大量的广泛而深入的研究. 至今这股波 及全世界的研究热潮没有丝毫减退, 每年都会涌现出很
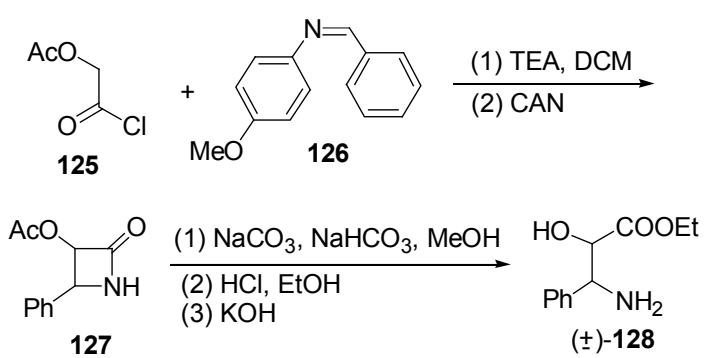
$\mathrm{H}_{2} \mathrm{O}, \mathrm{CAL}-\mathrm{B}$ i- $\mathrm{Pr}_{2} \mathrm{O}, 60^{\circ} \mathrm{C}$ $43 \%$, ee $>98 \%$ $\stackrel{\mathrm{N} \mathrm{H}_{2}}{ }$<smiles>O=C(O)[C@@H](O)Cc1ccccc1</smiles> $\longleftarrow \frac{18 \% \mathrm{HCl}}{4}$
$-129$

$\mathrm{H}_{2} \mathrm{O}$, Lipase PS-IM $i-\mathrm{Pr}_{2} \mathrm{O}, 50^{\circ} \mathrm{C}$ $\checkmark 7 \%$, ee $>99 \%$<smiles>CCOC(=O)[C@H](O)[C@H](N)c1ccccc1</smiles>

$(-)-128$

\section{Scheme 22}

多新颖的高水平的研究成果. 近年来, 在紫杉醇及其类 似物的合成研究中, 出现了很多巧妙而高效的策略, 例 如各种串联反应的应用、转化合成和不对称催化等. 另 外, 紫杉醇的结构修饰和活性研究也在不断扩大和深 入, 被开发的新型紫杉醇类似物日益增多, 围绕紫杉醇 的应用也越来越广泛. 紫杉醇在未来的药物治疗、化学 研究和生物技术领域必将继续占据着举足轻重的地位.

\section{References}

[1] Ganesh, T.; Norris, A.; Sharma, S.; Bane, S.; Alcaraz, A. A.; Snyderc, J. P.; Kingston, D. G. I. Bioorg. Med. Chem. 2006, 14, 3447.

[2] Rowinsky, E. K. Annu. Rev. Med. 1997, 48, 353.

[3] Schiff, P. B.; Fant, J.; Horwitz, S. B. Nature 1979, 277, 665.

[4] For total synthesis of taxol, see: (a) Nicolaou, K. C.; Yang, Z.; Liu, J. J.; Ueno, H.; Nantermet, P. G.; Guy, R. K.; Claiborne, C. F.; Renaud, J.; Couladouros, E. A.; Paulvannan, K.; Sorensen, E. J. Nature 1994, 367, 630.

(b) Nicolaou, K. C.; Ueno, H.; Liu, J. J.; Nantermet, P. G.; Yang, Z.; Renaud, J.; Paulvannan, K.; Chadha, R. J. Am. Chem. Soc. 1995, 117, 653 .

(c) Masters, J. J.; Link, J. T.; Snyder, L. B.; Young, W. B.; Danishefsky, S. J. Angew. Chem., Int. Ed. 1995, 34, 1723.

(d) Danishefsky, S. J.; Masters, J. J.; Young, W. B.; Link, J. T.; Snyder, L. B.; Magee, T. V.; Jung, D. K.; Isaacs, R. C. A.; Bornmann, W. G.; Alaimo, C. A.; Coburn, C. A.; Grandi, M. J. D. J. Am. Chem. Soc. 1996, 118, 2843.

(e) Holton, R. A.; Somoza, C.; Kim, H. B.; Liang, F.; Biediger, R. J.; Boatman, P. D.; Shindo, M.; Smith, C. C.; Kim, S.; Nadizadeh, H.; Suzuki, Y.; Tao, C.; Vu, P.; Tang, S.; Zhang, P.; Murthi, K. K.; Gentile, L. N.; Liu, J. H. J. Am. Chem. Soc. 1994, 116, 1597.

(f) Holton, R. A.; Kim, H. B.; Somoza, C.; Liang, F.; Biediger, R. J.; Boatman, P. D.; Shindo, M.; Smith, C. C.; Kim, S. J. Am. Chem. Soc. 1994, 116, 1599.

(g) Wender, P. A.; Badham, N. F.; Conway, S. P.; Floreancig, P. E.; Glass, T. E.; Houze, J. B.; Crauss, N. E.; Lee, D.; Marquess, D. G.; McGrane, P. L.; Meng, W.; Natchus, M. G.; Shuker, A. J.; Sutton, J. C.; Taylor, R. E. J. Am. Chem. Soc. 1997, 119, 2757.

(h) Mukaiyama, T.; Chiina, I.; Iwadare, H.; Saitoh, M.; Nishimura, 
T.; Ohkawa, N.; Sakoh, H.; Nishimura, K.; Tani, Y. I.; Hasegawa, M.; Yamada, K.; Saitoh, K. Chem. Eur. J. 1999, 5, 121.

(i) Morihira, K.; Hara, R.; Kawahara, S.; Nishimori, T.; Nakamura, N.; Kusama, H.; Kuwajima, I. J. Am. Chem. Soc. 1998, 120, 12980.

(j) Kusama, H.; Hara, R.; Kawahara, S.; Nishimori, T.; Kashima, H.; Nakamura, N.; Morihira, K.; Kuwajima, I. J. Am. Chem. Soc. 2000, 122, 3811 .

[5] For a formal synthesis of taxol, see: Doi, T.; Fuse, S.; Miyamoto, S.; Nakai, K.; Sasuga, D.; Takahashi, T. Chem. Asian J. 2006, 1, 370 .

[6] For selected reviews on the synthesis of taxol, see: (a) Wender, P. A.; Natchus, M. G.; Shuker, A. J. Taxol: Science and Applications, Ed.: Suffness, M., CRC, New York, 1995, pp. 123 187 .

(b) Narasaka, K.; Iwasawa, N. Chemtracts 1998, 11, 23.

(c) Tang, S. H. Frontiers of Biotechnology \& Pharmaceuticals, Vol. 1, Science Publisher, New York, 2000, pp. 336 348.

(d) Kingston, D. G. I.; Jagtap, P. G.; Yuan H.; Samala, L. Prog. Chem. Org. Nat. Prod. 2002, 84, 53.

(e) Han, G. D.; Ding, J. P.; Xie, L. Chin. J. Org. Chem. 1993, 13, 337 (in Chinese).

(韩广甸, 丁炬平, 谢蓝, 有机化学, 1993, 13, 337.)

(f) Fang, W. S. Chin. Pharm. J. 1994, 29, 259 (in Chinese).

(方唯硕, 中国药学杂志, 1994, 29, 259.)

(g) Huang, H. M.; Li, Y. Z. Chin. J. Synth. Chem. 1998, 6, 238 (in Chinese).

(黄化民, 李叶芝, 合成化学, 1998, 6, 238.)

(h) Xu, L.; Wang, F. P. Chin. J. Org. Chem. 2001, 21, 493 (in Chinese).

(徐亮, 王锋鹏, 有机化学, 2001, 21, 493.)

(i) Chen, Q. H.; Wang, F. P. Nat. Prod. Res. Dev. 2001, 13, 88 (in Chinese).

(陈巧鸿, 王锋鹏, 天然产物研究与开发, 2001, 13, 88.)

(j) Yan, J. Q. Jiangsu Chem. Ind. 2005, 33, 46 (in Chinese).

(阎家麒，江苏化工, 2005, 33, 46).

(k) Lin, B. R.; Feng, X.; Xu, F. Chem. Reag. 2006, 28, 461 (in Chinese).

(吝保瑞, 冯霞, 徐芳, 化学试剂, 2006, 28, 461.)

(1) Li, L. G.; Wu, M.; Shi, Q. W. Nat. Prod. Res. Dev. 2008, 20, 1104 (in Chinese).

(李力更, 吴明, 史清文, 天然产物研究与开发, 2008, 20, 1104.) (m) Zhao, Y.; Yao, Z. J. In Highlights of Total Synthesis of Natural Products-Terpenes, Eds.: Wu, Y. L.; He, Z. L., Science Press, Beijing, 2010, pp. 1 38 (in Chinese).

(赵昱, 姚祝军, 天然产物全合成荟萃——萜类, 吴毓林, 何子 乐主编, 科学出版社, 北京, 2010, pp. 1 38).

[7] Utsugi, M.; Kamada, Y.; Nakada, M. Tetrahedron Lett. 2008, 49, 4754.

[8] (a) Shimada, Y.; Nakamura, M.; Suzuka, T.; Matsui, J.; Tatsumi, R.; Tsutsumi, K.; Morimoto, T.; Kurosawa, H.; Kakiuchi, K.; Matsukubo, T. Tetrahedron Lett. 2003, 44, 1401.

(b) Enomoto, T.; Morimoto, T.; Ueno, M.; Matsukubo, T.; Shimada, Y.; Tsutsumi, K.; Shirai, R.; Kakiuchi, K. Tetrahedron 2008, 64, 4051.

[9] Aldegunde, M. J.; Castedo, L.; Granja. J. R. Org. Lett. 2008, 10, 3789.

[10] Zou, C. L.; Cai, L.; Ji, H.; Xie, G. B.; Wang, F. P.; Jian, X. X.; Song, L.; Liu, X. Y.; Chen, D. L.; Chen. Q. H. Tetrahedron 2008, 64, 7594.

[11] Goldring, W. P. D.; Pattenden, G.; Rimmington, S. L. Tetrahedron 2009, 65, 6670.

[12] Petrignet, J.; Boudhar, A.; Blond, G.; Suffert, J. Angew. Chem., Int. Ed. 2011, 50, 3285.

[13] Mendoza, A.; Ishihara, Y.; Baran, P. S. Nat. Chem. 2012, 4, 21.

[14] Kaliappan, K. P.; Ravikumar, V.; Pujari, S. A. J. Chem. Sci. 2008, 120, 205.

[15] Ma, C.; Schiltz, S.; Goff, X. F. L.; Prunet, J. Chem. Eur. J. 2008, 14, 7314 .

[16] Brémond, P.; Audran, G.; Monti, H. J. Org. Chem. 2008, 73, 6033.

[17] Serizawa, T.; Miyamoto, S.; Numajiri, Y.; Fuse, S.; Doi, T.; Takahashi, T. Tetrahedron Lett. 2009, 50, 3408.

[18] Ma, C.; Schiltz, S.; Prunet, J. Collect. Czech. Chem. Commun. 2011, 76, 1579.

[19] Mustafa, E.; Necdet, C. ARKIVOC 2009, xii, 153.

[20] Dziedzic, P.; Schyman, P.; Kullberg, M.; Córdova, A. Chem. Eur. J. 2009, 15, 4044.

[21] Hernández, L. M.; Soderquist, J. A. Org. Lett. 2009, 11, 2571.

[22] Qian, Y.; Xu, X. F.; Jiang, L. Q.; Prajapati, D.; Hu, W. H. J. Org. Chem. 2010, 75, 7483.

[23] (a) Forró, E.; Fülöp, F. Tetrahedron: Asymmetry 2010, 21, 637. (b) Forró, E.; Fülöp, F. Eur. J. Org. Chem. 2010, 3074. 